\section{CHILD PROTECTION}

\section{G31 SUBDURAL HAEMORRHAGE (SDH) IN CHILDREN OVER 2 YEARS}

\section{Russell CF, Smith HC Royal Manchester Children's Hospital}

Aims: To determine the aetiology, pattern of associated injuries and clinical course of children aged 2-16 years admitted with subdural haemorrhage (SDH).

Method: Records of 33 children (23 boys) aged 2-16 years, identified through Hospital Activity Analysis (HAA) codes, were reviewed retrospectively.

Results: Age at presentation showed a bimodal distribution with peaks at 3 years and $10-12$ years.

For all 33 children, there was a clear history of major head trauma prior to admission. 21 were injured in road accidents, 7 suffered serious falls, 3 tripped and 2 were assaulted.

All had radiological evidence of acute SDH. 29/33 SDH's were unilateral. 24 had a skull fracture and 13 of these also had cerebral oedema and/or contusion.

$18 / 33$ were comatose on admission and $25 / 33$ were admitted to ITU. There were 9 deaths.

Conclusions: SDH in childhood is usually unilateral and presents after acute injury by impact. This is in contrast to infant $\mathrm{SDH}$ which is usually bilateral and results from a combination of rotational and deceleration forces. Paediatricians managing SDH in infants should be aware of the types of trauma causing $\mathrm{SDH}$ in childhood.

\section{G32 FAMILY AND PERPETRATOR PATTERNS IN NON-} ACCIDENTAL HEAD INJURY

S Sivaramakrishnan, C Lazaro Lindisfarne Centre, RVI, Newcastle upon Tyne

Aims: To examine family and perpetrator patterns in 59 infants with non-accidental head injury.

Methods: Retrospective case review of suspected non-accidental head injuries referred to $\mathrm{CL}$ over a period of 8 years.

Results: 59 victims were identified, of which $72 \%$ were male and $35 \%$ under 8 weeks of age. $69 \%$ had brain trauma including subdural haemorrhages, $50 \%$ had skull fractures, $40 \%$ multiple fractures. $12 \%(7)$ infants died.

$71 \%$ of mothers were under 25 years of age. Pregnancy was unplanned in $66 \%$. Infants were first born in $53 \%$.

The male parent figure was not the natural father in 18 cases $(30.5 \%)$

28 parents $(47.4 \%)$ had taken the infant to the A\&E Department on more than 2 occasions before the ultimate incident. Violence between partners was reported in 12 cases $(20 \%)$ and mental health problems in 21 families $(35.5 \%)$. $83 \%$ of the infants were considered to be injured by the father figure, $59.3 \%$ by the biological father and $23.7 \%(14)$ by the non-biological father figure. The mother was suspected in $10(16.9 \%)$.

In 27 cases (45\%), the mother was absent from the home at the time of the alleged incident.

Conclusions: This study confirms that repeated attendance at A\&E of young infants should be of serious concern and a history of family violence and psychiatric disturbance should be actively sought. Male parental figures are most often involved in deliberate head trauma.

\section{G33 ARE OUR SCREENING CRITERIA FOR FAILURE TO THRIVE} (FTT) APPROPRIATE?

Heycock E, Rudolf MCJ, O'Brien L Postgraduate Medicine, North Staffordshire Hospitals. Belmont House, Leeds Community and Mental Health Trust

Background and aims: For research and screening purposes, failure to thrive is usually defined as weight $<2^{\text {nd }}$ or $3^{\text {rd }}$ centile, and/or crossing down 2 centiles. In the course of a study on FTT, the investigators became aware that many children were included whose growth curves would not have aroused their concern. They therefore devised a study to address whether there was a discrepancy between conventional criteria and their concerns as clinicians.

Methods: Two consultant paediatricians experienced in FTT, blindly and independently reviewed all the growth charts of children who had been screened as FTT through child health surveillance. They each categorised the charts as requiring: a) paediatric evaluation, b) health visitor review, or c) routine surveillance. They repeated their assessments again 3 months after the date the children were screened in as FTT.

Results: 174 charts were examined. At the time of identification, 48 children $(28 \%)$ were considered to need paediatric assessment, $33(19 \%)$ review by the health visitor, and $93(53 \%)$ as normal. At the followup interval (after no intervention) only $27(15 \%)$ children were deemed to require paediatric input, while $147(85 \%)$ were now agreed to be normal. Close agreement was attained between the 2 paediatricians on both occasions.
Conclusions: Using conventional criteria for screening children for FTT, two independent experts agreed that only a minority of children identified had concerning growth charts. This finding was supported by the fact that $85 \%$ children 3 months later had shown satisfactory growth. This report suggests that our current criteria for identifying FTT needs to be revisited and revised. We are likely to be identifying many normally growing children, and arousing anxieties in doing so.

\section{G34 PROBABILITIES OF BRUISE PATTERNS BEING DUE TO ABUSE: A CASE CONTROL STUDY}

Guildea Z, Kontos K, Kemp A M, Dunstan F D, Sibert JR Family Studies Research Centre, University of Wales College Of Medicine, Department of Child Health, Academic Centre, Llandough Hospital, Penarth, Vale of Glamorgan, CF64 2XX

Aims of Study: Although bruising is the commonest feature of physical abuse in children, there is only a small evidence base for attempting to diagnose abuse from a particular bruise pattern. The aims of this study, therefore, were to develop a method for recording bruise patterns, to compare bruise patterns in groups of abused and control children and to investigate their use in identifying abused children.

Methods: We developed a method of recording bruise patterns using the maximum dimension and any specific pattern of each bruise in 12 areas of the body. We collected information on the bruise pattern of 147children attending the Llandough Children Centre from 1992-8 and later shown, at a Case Conference or other multidisciplinary meeting, to have been abused. We also collected information on the bruise pattern of 199 control children attending the Centre for reasons other than abuse.

Results: The method of recording bruises was easy to use and produced mathematically analysable results. Different regions gave very different discriminatory power. In 8 of the 12 areas of the body, the cases had significantly more bruises than the controls $(p<0.0001)$. Numbers of bruises on the limbs were not significantly different between the two groups. Numbers in different regions were not independent, so they had to be combined for modelling purposes.

Conclusions: Our method of recording bruise patterns can produce information which, by using Bayes' Theorem, could aid the diagnosis of whether the whole pattern of injuries is due to abuse or not. The bruising pattern on the head and neck, or on the trunk, is very useful for discriminating between abused and non-abused children; bruising on the limbs may not add significantly to the power for identifying abuse in an individual child. 